\title{
Cloud droplet effective radius from spaceborne polarization measurements
}

\author{
François-Marie Bréon \\ Laboratoire de Modélisation du Climat et de l'Environnement, Commissariat à l'Energie Atomique, Gif sur Yvette, \\ France

\section{Philippe Goloub} \\ Laboratoire d'Optique Atmosphérique, Université des Sciences et Techniques de Lille, Villeneuve d'Ascq, France
}

\begin{abstract}
The spaceborne POLDER instrument provided the first quantitative measurements of the Earth reflectance polarization characteristics. Many POLDER images of polarized light show cloudbow type features over cloud fields for scattering angles between 150 and $170^{\circ}$. This unexpected observation is attributed to the polarized radiance generated by single scattering by cloud droplets. It shows that, in many cases, the cloud droplet size distribution is very narrow. The multidirectional polarized radiance measurements can be inverted for an accurate estimate of the cloud droplet radius.
\end{abstract}

\section{Introduction}

The POLDER instrument (Deschamps et al., 1994) was launched on the ADEOS platform in August 1996. Its measurement principle is based on a bidimensional CCD detector, a rotating wheel which carries optical filters and polarisers and a wide field of view optics. At present, POLDER is the only spaceborne instrument which provided quantitative measurements of the reflected sunlight polarization characteristics. Unfortunately, due to electrical failure, the ADEOS platform died on June 30th, 1997. Nevertheless, eight months of measurements have been acquired which allow new investigations on the usefulness of polarization and directionality for Earth remote sensing. In this paper, we show unanticipated observations in polarized light. Further investigation demonstrate that, in favorable conditions, the cloud droplet radius can be retrieved from POLDER measurements with a very high accuracy.

\section{Observation}

Figure 1 is a three color composite (Blue : $0.44 \mu \mathrm{m}$; Green : $0.67 \mu \mathrm{m}$; Red: $0.86 \mu \mathrm{m}$ ) of the radiance measured by the instrument CCD detector over a $1600 \times 2200 \mathrm{~km}^{2}$ area located across the west coast of Africa, south of the Equator. The image center location is approximately $\left(20^{\circ} \mathrm{S}, 10^{\circ} \mathrm{E}\right)$. The black/white lines overlaid on the figure indicate the scattering angle in $10^{\circ}$ increments (angle between the solar incident and the viewing directions) as well as the principal plane. The top figure shows the total radiance and is similar to what would be observed with an instrument without polarization capabilities. On the right side of the image

Copyright 1998 by the American Geophysical Union.
(East) is the African continent, with more vegetation to the North (top) which is evidenced by the redish color (larger reflectance at $0.86 \mu \mathrm{m}$ ). A few cloud fields appear as white areas over the land. On the left side of the figure is the Atlantic ocean, which is covered by a variable amount of clouds. This area is well known for a very high occurrence of stratocumulus.

The bottom image corresponds to the exact same area, but displays the perpendicular component of the polarized radiance. In the Stokes vector representation of radiance $(\mathrm{I}, \mathrm{Q}, \mathrm{U}, \mathrm{V})$, it is the second component of the vector $(\mathrm{Q})$ where the reference direction is the plane of scattering (defined by the solar and viewing directions). The white band which follows the $140^{\circ}$ line of scattering angle corresponds to a maximum in the polarized radiance around this direction, which is characteristic of water droplets radius larger than the wavelength (Goloub et al., 1994). The intense cloudbow along the $140^{\circ}$ scattering angle line is an unambiguous indication of the presence of liquid water droplets at the cloud top. For larger scattering angles, color bands indicate a scattering process which is highly directional and spectrally dependent. This is peculiar since, in the spectral range of interest $(0.44-0.86 \mu \mathrm{m})$, cloud reflectances are usually expected to be roughly white.

To investigate further this phenomenon, we present in Fig. 2 the same image (only the polarized radiance is shown), but where the three spectral bands have been separated. The same general features are apparent. In the $140-170^{\circ}$ range, oscillations of the polarized reflectance as a function of the scattering angle are apparent. Note that the number of oscillations vary with the spectral band. There are about twice as many in the blue band $(0.44 \mu \mathrm{m})$ than in the near infrared $(0.86 \mu \mathrm{m})$. The difference in the position of the maxima and minima between the spectral bands explains the "cloudbow" features displayed in Fig. 1.

\section{Interpretation}

Radiative transfer simulations show that single scattering provides the main contribution to the polarized radiance. Multiple scattering polarization is very small (Hunsen, 1971). Assuming a plane parallel cloud, no absorption, and no other scattering processes, the single scattering contribution to the polarized reflectance is :

$$
R_{p}^{s s}=\frac{1-\exp \left[-\tau\left(1 / \mu_{s}+1 / \mu_{v}\right)\right]}{4\left(\mu_{s}+\mu_{v}\right)} P_{P}(\gamma)
$$



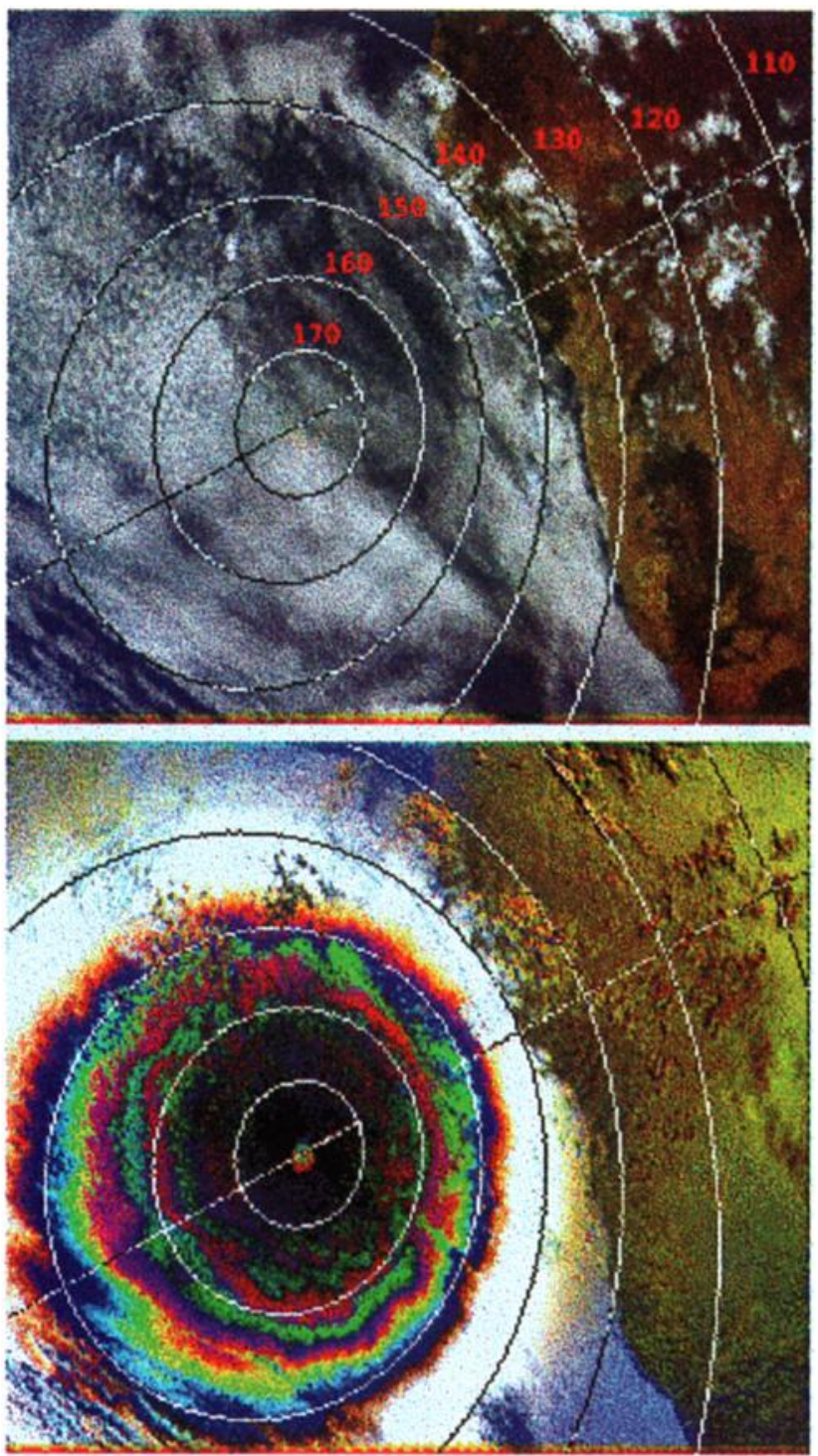

Figure 1. Three color composite (Blue : $0.43 \mu \mathrm{m}$, Green : 0.67 $\mu \mathrm{m}$, Red : $0.86 \mu \mathrm{m}$ ) of POLDER measurements acquired by the CCD matrix over the Atlantic ocean and Southern Africa on Nov. 3rd, 1996. The top figure is for the total reflectance, whereas the bottom figure represents the polarized reflectance. The curved lines indicate the scattering angle in $10^{\circ}$ increments (smaller radius line is for $170^{\circ}$ ). The straight line is the principal plane. For each spectral band, the scale is from 0 to 0.8 in reflectance, and from 0.08 in polarized reflectance.

scattering phase function of the droplets, $\mu_{S}$ and $\mu_{v}$ are the cosines of the solar and zenith angles, respectively, and $\gamma$ is the scattering angle. In most cases, the cloud optical thickness is such that the exponential takes negligible values. Equation (1) then reduces to :

$$
R_{p}^{s s}=\frac{P_{P}(\gamma)}{4\left(\mu_{s}+\mu_{v}\right)}
$$

Therefore, the polarized reflectance measurement is directly linked to the polarized phase function. Such functions are calculated using the Mie theory. Fig. 3 shows the polarized phase function $P_{p}(\gamma)$ computed for several cloud droplet size distributions as (Deirmendjian, 1969 ) :


Figure 2. Same as in Fig. 1 but the three bands have been separated and only the polarized reflectance is shown. The spectral band is $0.44,0.67$ and $0.86 \mu \mathrm{m}$ from top to bottom. 


$$
n(r)=\left(\frac{r}{r_{\text {eff }}}\right)^{-3} \exp \left[\frac{1}{\sigma_{\text {eff }}}\left(\ln \left(\frac{r}{r_{\text {eff }}}\right)-\frac{r}{r_{\text {eff }}}+1\right)\right]
$$

where $n(r)$ is proportional to the droplet number concentration, and $r_{\text {eff }}$ (effective radius) and $\sigma_{\text {eff }}$ (effective variance) are two parameters which define the size distribution.

The polarized phase function shows local maxima and minima in the range $150-170^{\circ}$. Note that, as on the POLDER measurements, there are about twice as many oscillations at $0.44 \mu \mathrm{m}$ than at $0.86 \mu \mathrm{m}$ (Fig. 3a). As the size distribution broadens ( $\sigma_{e f f}$ increases), the amplitude of the oscillations decreases. For effective variances of 0.1 , they are smoothed out.

These results provide a simple interpretation of the observed cloudbow features. The observation of similar cloudbows over many large areas indicate that 1) an homogeneous cloud droplet size distribution is a frequent phenomenon, and 2) the size distribution over these areas is relatively narrow $\left(\sigma_{\text {eff }} \leq 0.05\right)$.

\section{Inversion}

To investigate further this phenomenon, and its implication for the remote sensing of cloud droplet radius, we
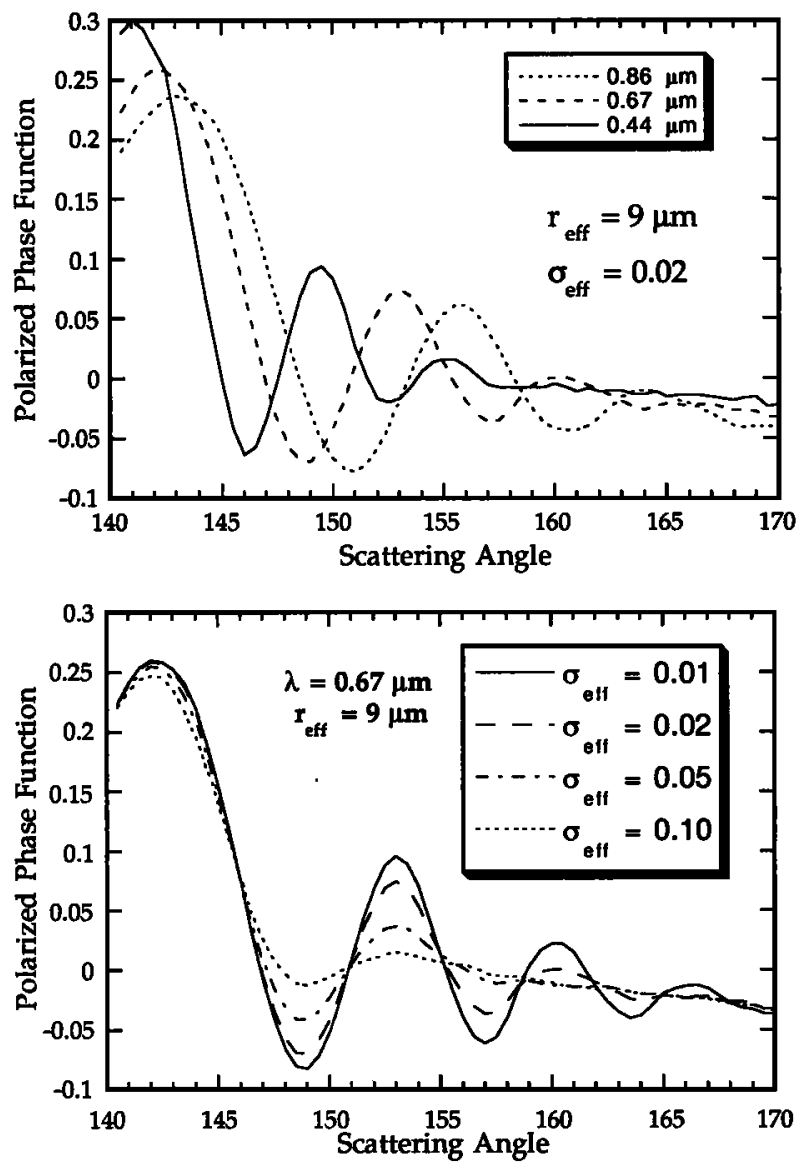

Figure 3. Polarized phase function $P p(\gamma)$ as a function of scattering angle for cloud droplet size distributions as in eq. (3). In Fig. 3a, we show how $P p(\gamma)$ varies with the wavelength. Fig. $3 \mathrm{~b}$ illustrates the variation of $P p(\gamma)$ with the size distribution variance.


Figure 4. Two examples of model-measurement comparisons after a successful inversion of cloud droplet effective radius from POLDER spaceborne measurements. In both cases, the dispersion $\sigma_{\text {eff }}$ is $\mathbf{0 . 0 2}$.

have separated the POLDER swath into $200 \times 200 \mathrm{~km}^{2}$ boxes. For each box, we make use of the directional capabilities of the POLDER instrument (each pixel is viewed from up to fourteen different geometries during a single satellite overpass). For a given pixel, the scattering angle sampling is on the order of $5^{\circ}$, which is not sufficient to observe the oscillations. The spatial agregation is therefore necessary, assuming homogeneity, because the various pixels in a given box are observed with slightly different viewing angles. A minimum size of $150 \times 150 \mathrm{~km}^{2}$ yields an homogeneous sampling of the scattering angles. However, if the cloud droplet distribution is heterogeneous within the area, the measurement variation with scattering angle is noisy, and the inversion is impossible.

The measurements are first corrected for Rayleigh scattering with the assumption that the cloud top height is about $1 \mathrm{~km}$ :

$R_{p}=R_{p}^{m e s} \exp \left[-\tau_{m}\left(\frac{1}{\mu_{s}}+\frac{1}{\mu_{v}}\right)\right]-R_{p}^{m o l}\left(\tau_{m}, \mu_{s}, \mu_{s}, \varphi\right)$

where $\tau_{m}$ is the molecular optical thickness above a theoretical $1 \mathrm{~km}$ high cloud top, and $R_{p}^{m o l}$, the result of radiative transfer simulations, is the polarized reflectance generated by molecular scattering in the atmosphere. A normalization for the viewing geometry [i.e. multiplication by $\left.4\left(\mu_{s}+\mu_{v}\right)\right]$ is then applied. In the single scattering approximation, the normalized measurements are roughly 
proportional to the polarized phase function. We then average all corrected measurements within a given box as a function of the scattering angle.

The polarized phase functions $P_{p}$ have been computed for a large number of size distributions defined by (3). The measurements are fitted by a function $F(\lambda, \gamma)$ where $\lambda$ is the wavelength :

$$
F(\lambda, \gamma)=a P_{p}\left(\gamma, \lambda, r_{e f f}, \sigma_{e f f}\right)+b \gamma+c
$$

In eq. (5), $a, b$ and $c$ are three parameters which are inverted through a least square procedure (minimization of the squared difference between the measurements and the function defined in (5), for a given range of scattering angle). "a" accounts for the cloud fractional coverage; $b \gamma+c$ accounts for the polarized radiance generated by aerosol above the cloud, inaccuracies in the molecular scattering correction and contributions from the glitter. The cloud droplet model which best fits the three wavelengths measurements for a range of scattering angle between $150^{\circ}$ and $170^{\circ}$ is retained. The inversion is successful if " $a$ " is greater than a threshold and the measurement-model RMS error is lower than another threshold.

Fig. 4 shows two examples of results which yield effective radius of $8 \mu \mathrm{m}$ and $10 \mu \mathrm{m}$. Note that position of the maxima and minima are very different for the two cases when the effective radii differ by only two microns. This is an indication of the sensitivity of the method to changes in effective droplet radius. The agreement between model and measurements is excellent. The position of the maxima is accurately reproduced by the model for the three bands. The agreement in Fig. $4 \mathrm{~b}$ is not as good for the $0.44 \mu \mathrm{m}$ band than for the two others. This may result from an inacurate correction of the molecular contribution to the polarized light which is much larger at this wavelength than at the others.

The agreement is not as good close to $145^{\circ}$ of scattering angle. This angle corresponds to the main cloudbow which shows a larger and wider maximum in the polarized phase function. We have made multiple scattering simulations of polarized light for plane parallel cloud using a code developed by Garcia and Siewert (1986). These simulations show that the single scattering approximation used in the inversion procedure (eq. 2 and eq. 5 ) is very accurate for local maxima in the range $145-170^{\circ}$ but underestimates the polarized radiance in the main cloudbow. This underestimation results from photons which are scattered with a scattering angle around $145^{\circ}$ and again with a very small angle (forward peak of the phase function), or the opposite. Such photons, which are not accounted for in the single scattering approximation, have a polarization similar to those single scattered and contribute to the polarized radiance around $145^{\circ}$. Because the secondary cloudbows are narrower in term of scattering angle, this double scattering process is smoothed out in the $150-170^{\circ}$ range.

\section{Discussion}

The observation of several cloudbows in the $150-170^{\circ}$ range of scattering angles is much easier in polarized light than in total radiance. For most clouds (optical thickness larger than $\approx 2$ ), the total reflected radiance is mostly generated by multiple scattering within the cloud. In such case, single scattering is a small contribution to the radiance and the relative amplitude of the reflectance variations with scattering angle is small (Spinhime and Nakajima, 1994). On the same scenes as those presented in this paper, we have not been able to measure a variation of the total radiance with a change in scattering angle which is significant with regard to the spatial variation (see Fig. 1a).

The proposed inversion method is applicable only if the droplet size distribution is relatively narrow. For broad size distributions, the phase function maxima and minima are smoothed out and the effective radius retrieval is impossible. Another limitation of the method is that it only probes a cloud layer of optical thickness less than about 1 located at the cloud top. On the other hand, this may be seen as an improvement to other methods based on the spectral signatures of clouds, for which the radius estimate is an indefinite average over the cloud layer. Nevertheless, for favorable conditions, the method is extremely sensitive and yields an unambiguous measurement of the cloud top effective radius. When the inversion is successful, the method also demonstrates that the size distribution is narrow.

The inversion method will now be applied to the full POLDER dataset. We will attempt a comparison of inverted radius with available in-situ measurements. We will also analyze the global distribution of retrieved droplet size in relation to atmospheric parameters such as aerosol loading estimates.

Acknowledgments. The results presented in this paper were obtained using data from CNES/POLDER onboard NASDA/ADEOS.

\section{References}

Deirmendjian, D. Electromagnetic scattering on spherical polydispersions, American Elsevier Publ. Co., New York, 1969.

Deschamps, P. Y., F. M. Brén, M. Leroy, A. Podaire, A. Bricaud, J. C. Buriez and G. Seze, The POLDER mission: Instrument characteristics and scientific objectives. IEEE Transactions on Geoscience and Remote Sensing, 32, 598-615, 1994.

Garcia, R.D.M., and C.E. Siewert; A generalized spherical harmonics solution for radiative transfer models that include polarization effects, J. Quant. Spec. Rad. Transfer, 36, 401-423, 1986.

Goloub P., J.L. Deuzé, M. Herman, Y. Fouquart, and P.Y. Deschamps, Analysis of the POLDER Airborne Instrument Observations over Cloud Covers, IEEE Trans. Geosc. Rem. Sens., 32, 78-88, 1994.

Han, Q., W.B. Rossow and A.A. Lacis, Near-global survey of effective droplet radii in liquid water clouds using ISCCP data, J. Clim., 7, 465-497, 1994.

Hansen, J.E., Multiple scattering of polarized light in planetary atmospheres. Part II. Sunlight reflected by terrestrial water clouds, J. Atm. Sciences, 28, 1400-1426, 1971.

Spinhime, J.D. and T. Nakajima, Glory of clouds in the near infrared, Appl. Opt., 33, 4652-4662, 1994.

\footnotetext{
F.M. Bréon, CEA/DSM/LSCE, 91191 Gif sur Yvette, France. (e-mail : fmbreon@cea.fr)

$\mathrm{Ph}$. Goloub, Laboratoire d'Optique Atmosphérique, bat P5, USTL, 59655 Villeneuve d'Ascq, France.
}

(Received January 16, 1998, revised March 26, 1998, accepted April 3, 1998) 